\title{
Positional right hip and thigh pain
}

\author{
Taylor J. Stone • Jenny T. Bencardino • \\ Zehava S. Rosenberg
}

Published online: 13 December 2014

(C) ISS 2014

\section{Part II}

Diagnosis

Magnetic resonance (MR) images demonstrate a hernia sac in the infrapiriformis greater sciatic foramen containing nonobstructed bowel loops and exerting mass upon the sciatic nerve, which is displaced and flattened, but maintains normal signal intensity. There is no edema within the herniated mesentery and no evidence of bowel obstruction. Note the dependent fluid and nondependent gas within the bowel. Incidentally noted are numerous small marrow-replacing lesions within the sacrum in this patient with metastatic cancer.

The patient presented with right hip and thigh pain, which was accentuated by walking and relieved by lying supine. The pain was preventing her from performing activities of daily living. There was no evidence of muscle weakness or atrophy on physical examination. The patient was not considered a surgical candidate because of the extent of metastatic disease and her refusal of further treatment. She did report pain improvement with gabapentin therapy, which was initiated secondary to concerns of sciatic pain.

\section{Discussion}

Sciatic foramen hernias are rare hernias of the pelvis and can involve the greater or lesser sciatic foramen. A surgical review of sciatic hernias described 99 cases occurring between 1900

The case presentation can be found at doi:10.1007/s00256-014-2076-2

T. J. Stone $(\bowtie) \cdot J$. T. Bencardino $\cdot$ Z. S. Rosenberg

New York University Langone Medical Center, Hospital for Joint Diseases, New York, NY 10003, USA

e-mail: taylor.stone.md@gmail.com and 2008 [1]. Sciatic hernias have been described containing various gastrointestinal and genitourinary structures, including small bowel, omentum, Meckel's diverticulum, colon, ovary, fallopian tube, bladder, and ureter $[2,3]$. As a result of the various structures involved, presentations are widely variable. Half of all patients present with nonspecific hip pain and one third have a palpable mass [1]. Other presentations include sciatica, bowel obstruction, or urinary obstruction [1, 3-5]. In the case of bowel obstruction, perforation of strangulated bowel can result in abscess formation [6]. Given the presentation variability, sciatic foramen hernias may be encountered through different imaging modalities and protocols. The diagnosis of sciatic hernia has been made through small bowel barium studies, peritoneography, transgluteal ultrasound, computed tomography (CT), and MR [1].

In the acute setting, CT is generally the fastest and most readily available imaging modality. For an outpatient with metastatic cancer and hip pain, an MR is likely to be the ordered test. Therefore, the radiologist is likely to encounter the entity through one of these two modalities. The sciatic notch is divided in the greater and lesser sciatic foramina by the sacrospinous and sacrotuberous ligaments. The piriformis divides the greater sciatic foramen into the suprapiriform and infrapiriform spaces. Therefore, three types of sciatic foramen hernias are possible based on the opening through which they emerge. Suprapiriformis hernias course with the superior gluteal vessels and nerve. Infrapiriformis hernias course with the inferior gluteal vessels, sciatic nerve, and pudendal vessels. Lesser sciatic foramen hernias pass medial to the neurovascular bundle [1]. On CT or MR, a hernia appears as an encapsulated mass of bowel loops located deep to the gluteus maximus. As with all hernias, imaging findings typically include convergence and twisting of mesenteric vessels - or other herniated structures — at the hernia origin [7].

Dynamic ultrasound in standing position or while performing Valsalva maneuvers may also be used. One case 
has been described in which the hernia was only apparent on ultrasound with the patient standing, after having a falsenegative CT examination [8]. Furthermore, if the hernia extends distal to the gluteus maximus, a mass may be palpable, in which case the ultrasound probe may be placed directly on the mass. On all imaging modalities, care should be taken to determine the contents of the hernia sac. Hernias of the ureter represent a special category that generally mandates care by a urologist [1].

The diagnosis may be of particular importance for radiologists, particularly musculoskeletal or interventional radiologists, who perform CT- or ultrasound-guided diagnostic/ therapeutic perisciatic nerve, piriformis muscle, or hamstring muscle injections, as herniated pelvic viscera will be in close proximity to the target injection. A case report has described gluteal abscess after an intramuscular injection in a patient with a previously unknown sciatic foramen hernia, although no imaging was provided that showed the injection itself perforated the bowel [5].

Sciatic hernias with incarcerated or strangulated contents should be treated surgically as soon as possible; hernias that are uncomplicated and reducible should be treated after routine preparation [1]. Transabdominal, preperitoneal, or transgluteal approaches can be used for surgical sciatic hernia treatment, depending on the structures involved in the hernia sac and the urgency of the procedure [1]. Although these hernias are not common, they may be encountered by musculoskeletal radiologists, particularly in patients with right hip or sciatic nerve pain, and should be recognized as they often warrant surgical management.

Conflict of interest The authors declare that they have no conflict of interest.

\section{References}

1. Losanoff JE, Basson MD, Gruber SA, Weaver DW. Sciatic hernia: a comprehensive review of the world literature (1900-2008). Am J Surg. 2010;199(1):52-9.

2. Touloupidis S, Kalaitzis C, Schneider A, Patris E, Kolias A. Ureterosciatic hernia with compression of the sciatic nerve. Int Urol Nephrol. 2006;38(3-4):457-8.

3. Yu PC, Ko SF, Lee TY, Ng SH, Huang CC, Wan YL. Small bowel obstruction due to incarcerated sciatic hernia: ultrasound diagnosis. $\mathrm{Br}$ J Radiol. 2002;75(892):381-3.

4. Speeg JS, Vanlangendonck Jr RM, Fusilier H, Richardson WS. An unusual presentation of a sciatic hernia. Am Surg. 2009;75(11):113941.

5. Chitranjan MD, Kandpal H, Madhusudhan KS. Sciatic hernia causing sciatica: MRI and MR neurography showing entrapment of sciatic nerve. Br J Radiol. 2010;83(987):e65-6.

6. Servant CT. An unusual cause of sciatica. A case report. Spine (Phila Pa 1976). 1998; 23(19):2134-6.

7. Martin LC, Merkle EM, Thompson WM. Review of internal hernias: radiographic and clinical findings. AJR Am J Roentgenol. 2006;186(3):703-17.

8. Labib PL, Malik SN. Choice of imaging modality in the diagnosis of sciatic hernia. J Surg Case Rep. 2013; 2013(12). 